\title{
Posterior Chamber Phakic Intraocular Lenses for the Correction of Myopia: Factors Influencing the Postoperative Refraction
}

\author{
Pedro Serra ${ }^{1,2, *}$, Ángel Sánchez Trancón ${ }^{2}$, Oscar Torrado Sierra ${ }^{2}$, António Baptista ${ }^{3}$ \\ and Santiago Cerpa Manito ${ }^{2}$ \\ 1 Cataract and Refractive Surgery Unit, Ophthalmology Clinic Vista Sánchez Trancón, Calle la Violeta, \\ 06010 Badajoz, Spain \\ 2 Optics and Optometry Department, Instituto Superior de Educação e Ciências, Alameda das Linhas de Torres, \\ 1750-142 Lisboa, Portugal; sancheztrancon@sanheztrancon.com (Á.S.T.); \\ oscar.torrado.sierra@gmail.com (O.T.S.); santicerpa@yahoo.es (S.C.M.) \\ 3 Center of Physics, Campus de Gualtar, University of Minho, 4710-057 Braga, Portugal; \\ abaptista@fisica.uminho.pt \\ * Correspondence: pedro.serra@vistasncheztrancon.com
}

Citation: Serra, P.; Sánchez Trancón, Á.; Torrado Sierra, O.; Baptista, A.; Cerpa Manito, S. Posterior Chamber Phakic Intraocular Lenses for the Correction of Myopia: Factors Influencing the Postoperative Refraction. Optics 2021, 2, 292-305. https://doi.org/10.3390/opt2040028

Academic Editor: Thomas Seeger

Received: 30 September 2021 Accepted: 11 December 2021 Published: 16 December 2021

Publisher's Note: MDPI stays neutral with regard to jurisdictional claims in published maps and institutional affiliations.

Copyright: () 2021 by the authors. Licensee MDPI, Basel, Switzerland. This article is an open access article distributed under the terms and conditions of the Creative Commons Attribution (CC BY) license (https:// creativecommons.org/licenses/by/ $4.0 /)$.

\begin{abstract}
Posterior chamber phakic intraocular lens implantation is a refractive technique for the correction of myopia. This study aimed to identify those factors contributing to variability in postoperative refraction. Methods: This retrospective study evaluated 73 eyes (one eye per patient) implanted with myopic implantable collamer lenses (ICL). Eyes were divided into two groups, the low myopic group (LMG) (ICL > -9.5 DS) and the high myopic group (HMG) (ICL $\leq-9.5 \mathrm{DS})$, to compare the predictability, efficacy index, and postoperative refraction between groups. The association of postoperative refraction with anatomical, demographic, and optical features was assessed through correlation analysis and investigated using ray-tracing. Results: Postoperative refraction at 3 months for the whole group was close to emmetropia at $-0.02 \pm 0.37$ DS, the LMG tended toward myopia and the HMG, toward hyperopia. The results showed that $65 \%$ and $54 \%$ of the eyes had postoperative refraction of within \pm 0.25 DS, respectively, in the LMG and HMG, and in both groups, $100 \%$ were within \pm 1.00 DS. ICL implantation had a higher efficacy index in the HMG $(1.13 \pm 0.15)$ than in the LMG $(1.04 \pm 0.15)$. Postoperative refraction was positively associated with the vault $(R=0.408)$ and negatively correlated with ICL power $(R=-0.382)$. Conclusion: The predictability and effectiveness of ICL implantation is high in a wide range of myopias. Considering the expected vault and including accurate vertex measurements would contribute to improving the predictability of the results.
\end{abstract}

Keywords: phakic intraocular lens; implantable collamer lens; myopia; postoperative refraction; vault; vertex distance

\section{Introduction}

Currently, phakic posterior chamber intraocular lenses are an established form of refractive surgery that is widely used in the correction of myopia and astigmatism. The implantable collamer lens (ICL, STAAR Surgical, Monrovia, CA, USA) represents one of the many phakic intraocular lenses (IOL) available on the market, the material being based on a polymeric combination of hydroxyethyl methacrylate and porcine collamer [1]. Myopic ICLs have a plano-concave geometry, an optical zone of approximately 4.9 to $5.8 \mathrm{~mm}$ in diameter, and flexible plate haptics to support the ICL on the ciliary-sulcus complex [2]. An important advantage of these lenses over other forms of refractive surgery procedures is their applicability in high myopias, with ICL powers ranging from -18.00 to -1.00 diopter sphere (DS). In a recent review, the refractive results of 1905 myopic eyes implanted with ICLs showed that $\sim 91 \%$ of the eyes had a postoperative refractive error within $\pm 0.50 \mathrm{DS}$, and $\sim 99 \%$ were within \pm 1.00 DS. The results confirm the high refractive predictability 
of ICL surgery and, consequently, the high effectiveness and safety of the procedure [3], resulting in an improvement in the quality of life of the patients [4].

One important parameter is the predictability of the postoperative refraction with this surgery, depending on the difference between the postoperatively expected IOL position used for the power calculation and the postoperative, real IOL position. The difference between the expected and real positions changes the refractive predictability of the IOL, leading to a postoperative refractive error [5,6]. In the case of ICLs, the manufacturer uses a modified vertex formula to calculate the ICL power, which requires an estimation of the expected ICL position [7]. Considering that to maintain the eye's normal physiologic behavior, the posterior ICL surface should be set at a distance of between 250 and $750 \mu \mathrm{m}$ from the anterior crystalline lens, i.e., the vault [8], thus, a reasonable value to be used as a predictor of the ICL position is a vault of $500 \mu \mathrm{m}$. Nevertheless, a review of 2263 eyes reported that approximately $20 \%$ of the eyes had a vault outside the optimal range, with vaults ranging from 0 to $1300 \mu \mathrm{m}$, exposing the large variability of the vault [9]. This amplitude of vaults is expected to influence the refractive predictability of the ICL, especially when highly myopic eyes are implanted [10].

Another relevant aspect in the refractive outcomes of phakic IOL is the difference in the vertex distance, i.e., the distance between the spectacle plane and the corneal plane, used for refraction, and the distance assumed for the phakic IOL power calculation [11]. In myopic eyes, for instance, an underestimation of the vertex distance assumed for calculation leads to an overcorrection of myopia, which may result in postoperative hyperopic refraction. In highly myopic eyes, such as those commonly implanted with ICL, this assumes a special relevance as the tolerance of vertex error decreases with spectacle lens power.

The clinical results reported in moderately and highly myopic eyes implanted with ICL have shown good levels of predictability, effectiveness, and safety of the procedure [12]; therefore, the variability of the postoperative refraction has received little attention in the literature. Despite this evidence, small refractive errors, e.g., \pm 0.50 DS, may produce a decrease in the efficacy index (ratio of the postoperative uncorrected distance visual acuity (UDVA) to the preoperative corrected distance visual acuity (CDVA)) in the case of myopic refractive error or elicit an additional accommodative effort in the case of hyperopic refractive error. Additionally, phakic lens implantation requires the patient to elicit a stronger accommodative effort compared to correction with spectacles $[13,14]$. This, when associated with hyperopic postoperative refraction and a patient age close to the onset of presbyopia, or poor accommodative amplitude, may hamper near-vision tasks. Thus, the present study aimed to assess the influence of anatomical, refractive, and ICL-related parameters as predictors of postoperative refraction.

\section{Patients and Methods}

This study is a retrospective cases series comprising 73 eyes of 73 patients implanted with a spherical ICL for the correction of myopia (EVO-V4c, STAAR Surgical AG, Nidau, Switzerland). The patients were operated on between 2012 and 2018 in the Ophthalmology Clinic of Vista Sánchez Trancón (Badajoz, Spain) by one of the authors (S.C.M.). All patients included in this study were selected from the clinic's database using the following inclusion criteria: myopia lower than -20.00 DS, refractive astigmatism lower than -0.75 diopter cylinder (DC), internal anterior chamber depth (ACD) $\geq 2.8 \mathrm{~mm}$, and endothelial cell count $\geq 2000$ cells $/ \mathrm{mm}^{-2}$ (Topcon SP-2000P, Topcon Corporation, Tokyo, Japan). Eyes with previous corneal refractive surgery, the presence of crystalline lens opacities, evidence of amblyopia associated with causes other than a refractive error (e.g., strabismus), and retinal abnormalities hampering central vision were excluded from the analysis. This research adhered to the tenets of the Declaration of Helsinki and gained ethical approval from the local ethics committee (Comité Ético de Investigación Clínica de Badajoz, Spain). Due to the retrospective nature of the study, the informed consent criterion was waived. 


\subsection{Preoperative and Postoperative Protocol}

Preoperatively, patients had a full ophthalmologic examination, which included presenting visual acuity (VA) using a Snellen chart, subjective refraction, cycloplegic autorefraction (Topcon KR-8900, Topcon Corporation, Tokyo, Japan), and corrected distance VA (CDVA). Visual acuities, measured with a decimal scale, were transformed into logMAR units [15]. The subjective refraction was performed using a trial frame, with the spherical lenses placed in the frontward cells of the trial frame. The spherical component was determined through the fogging method, with the endpoint determined by the most positive lens enabling the best distance VA; the cylinder component was refined using the Jackson cross-cylinder method. Subjective refraction was assumed to have been performed with the spectacle plane distanced $12.0 \mathrm{~mm}$ from the corneal plane, i.e., vertex distance. The autorefraction readings were reported for a $12.0 \mathrm{~mm}$ vertex distance. Anterior segment anatomy was evaluated using a slit-lamp, intraocular pressure was measured with a Goldman tonometer, and the retina was inspected by indirect ophthalmoscopy. The biometric assessment of the anterior segment (Pentacam, OCULUS, Optikgeräte GmbH, Wetzlar, Germany) comprised the measurement of the central corneal thickness (CCT), horizontal visible iris diameter, i.e., the white-to-white (WTW), simulated central keratometry (Sim K), corneal astigmatism (CA), and ACD. The ICL lens size and dioptric power were determined by using the manufacturer's online calculation and ordering system (OCOS $\left.{ }^{\mathrm{TM}}\right)$. A $12.0 \mathrm{~mm}$ vertex distance was used to compute the ICL power through a modified vertex formula and the ICL power was chosen according to target emmetropia. The OCOS ${ }^{\mathrm{TM}}$ provides an estimation of the postoperative spherical-cylinder refraction, here defined as postoperative predicted refraction.

At three months postoperatively, the ICL position was assessed using anterior segment optical coherence tomography (AS-OCT) (Visante, Zeiss Meditec AG, Jena, Germany). The central vault was measured using the central vault line caliper by placing the line between the posterior ICL surface and the anterior crystalline lens surface apex (Figure 1). The AS-OCT imaging was performed under dim lighting conditions, with the patient fixating on the device's in-built fixation target. Uncorrected distance VA was measured with the same VA chart as was used preoperatively and autorefraction was performed using the same autorefractor. The postoperative spherical-cylinder autorefraction was defined as postoperative manifest refraction.

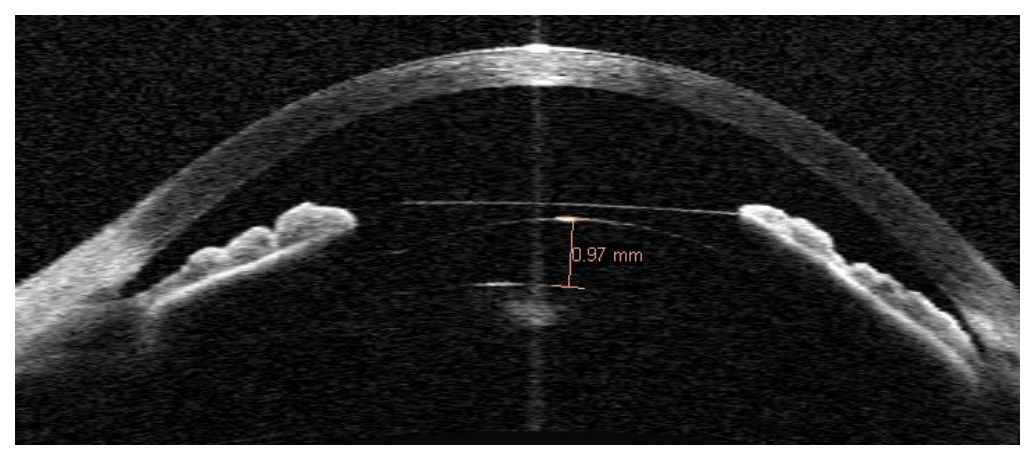

Figure 1. Anterior segment optical coherence tomography of an eye, implanted with a myopic ICL, exhibiting a $970 \mu \mathrm{m}$ vault.

\subsection{Surgical Procedure}

The surgery was performed under local anesthesia using $2 \%$ intracameral preservativefree lidocaine (B. Braun ${ }^{\circledR} 20 \mathrm{mg} / \mathrm{mL}$ ) and the anterior chamber was filled with viscoelastic ( $2 \%$ methylcellulose, Medicontur, Zsámbék, Hungary). The ICL was introduced through a $3.2 \mathrm{~mm}$ temporal clear corneal incision, using the manufacturer's injector cartridge (STAAR Surgical Co., Monrovia, CA, USA). After placing the ICL, the viscoelastic was aspirated from the anterior chamber. Finally, a diluted antibiotic solution (ceftazidime $50 \mathrm{mg} / \mathrm{mL}$ and vancomycin $50 \mathrm{mg} / \mathrm{mL}$ ) was injected. After surgery, antibiotic (Exocin ${ }^{\circledR}$, ofloxacin 
$3 \mathrm{mg} / \mathrm{mL}$ ), corticoid (Predforte ${ }^{\circledR}$, prednisolone acetate $10 \mathrm{mg} / \mathrm{mL}$ ), and a non-steroidal anti-inflammatory (Voltaren ${ }^{\circledR}$, diclofenac sodium $1 \mathrm{mg} / \mathrm{mL}$ ) drugs were prescribed four times a day for three weeks.

\subsection{Clinical Data Analysis}

Differences in postoperative refraction were initially tested by dividing the sample into two myopic groups, a low myopic group (LMG) comprising ICL powers $>-9.50$ DS and a high myopic group (HMG) comprising ICL powers $\leq-9.5 \mathrm{DS}$. The $-9.50 \mathrm{DS}$ cut-off was chosen since it was close to the mean ICL power of the sample, allowing for a balance in the number of eyes in both groups. Non-parametric Mann-Whitney analysis was used separately for seeking differences between the following variables: demographic (age), refractive (preoperative refraction, postoperative predicted refraction, and postoperative manifest refraction), anatomical (CCT, WTW, Sim K, CA, ACD), and ICL-related (ICL power, vault). Bonferroni correction was applied to account for multiple comparisons. The associations between postoperative refraction with the aforementioned variables were determined using bivariate correlation analysis. For the sake of analysis of the refractive data, the refraction measurements were transformed from the spherical-cylinder form to the spherical equivalent form (SE) using the equation: $\mathrm{SE}=$ sphere $+0.5 \times$ cylinder. The postoperative SE used in the correlation analysis was calculated as the postoperative manifest SE, minus the postoperative predicted SE. Differences in correlations between LMG and HMG were tested using a univariate analysis of variance. Statistical analysis was performed using SPSS $\left(\mathrm{IBM}^{\circledR}\right.$, SPSS $^{\circledR}$ Statistics, version 23).

\subsection{Paraxial Ray-Tracing}

The association between postoperative refraction and the vault was investigated using paraxial ray-tracing by applying the Le Grand full theoretical model eye [16], with the inclusion of two refractive surfaces representative of an ICL (Table 1).

Table 1. Le Grand full theoretical model eye details.

\begin{tabular}{|c|c|c|c|}
\hline \multicolumn{2}{|l|}{ Parameter } & \multicolumn{2}{|l|}{ Refractive Index } \\
\hline Spectacle Refraction & Variable & & \\
\hline Vertex Distance $(\mathrm{mm})$ & 12.0 & Air & 1.0 \\
\hline Anterior Corneal Radius (mm) & 7.80 & & \\
\hline Central Corneal Thickness (mm) & 0.55 & Cornea & 1.3771 \\
\hline Posterior Corneal Radius (mm) & 6.50 & & \\
\hline \multicolumn{4}{|l|}{ Cornea Endothelium to Crystalline Lens } \\
\hline Anterior Surface Distance (Anterior & 3.05 & Aqueous humor & 1.3374 \\
\hline \multicolumn{4}{|l|}{ Chamber Depth) (mm) } \\
\hline Crystalline Lens Anterior Radius (mm) & 10.20 & & \\
\hline Crystalline Lens Thickness (mm) & 4.00 & Crystalline lens & 1.420 \\
\hline Crystalline Lens Posterior Radius (mm) & -6.00 & & \\
\hline Vitreous Body Length (mm) & Variable & Vitreous body & 1.3360 \\
\hline \multicolumn{4}{|c|}{ Model Eye with Refractive Surfaces Representative of an ICL } \\
\hline $\begin{array}{l}\text { Cornea Endothelium to ICL Anterior } \\
\text { Surface Distance (mm) }\end{array}$ & 2.32 & Aqueous Humor & 1.3374 \\
\hline ICL Anterior Radius of Curvature (mm) & $\infty$ & & \\
\hline ICL Thickness (mm) & 0.23 & ICL Refractive Index & 1.442 \\
\hline ICL Posterior Radius of Curvature (mm) & Variable & & \\
\hline $\begin{array}{l}\text { ICL Posterior Surface to Crystalline Lens } \\
\text { Anterior Surface Distance, i.e., Vault (mm) }\end{array}$ & 0.50 & Aqueous humor & 1.3374 \\
\hline
\end{tabular}

The model eye can be described as a system matrix $(S)$, consisting of the product of the refraction $(R)$ and translation $(T)$ matrices (Equations (1)-(3)), representative of the refraction throughout various refractive surfaces $(m)$ with dioptric power $(P)$, and the translation throughout the various interspaces, with thickness, $d$, and refractive index, 
$n$ [17]. The matrix system was implemented in Matlab (Matlab ${ }^{\circledR}$ R2018a, The Mathworks Inc., Natick, MA, USA).

$$
\begin{gathered}
S=R_{m} T_{m-1, m} R_{m-1} T_{m-2, m-1} \ldots R_{2} T_{1,2} R_{1} \\
R=\left[\begin{array}{cc}
1 & -P \\
0 & 1
\end{array}\right] \text { with } P=\frac{n_{m+1}-n_{m}}{r_{m}} \\
T=\left[\begin{array}{ll}
1 & 0 \\
\frac{d}{n} & 1
\end{array}\right]
\end{gathered}
$$

The matrix $(S)$ of an eye implanted with a phakic IOL is described from the spectacle plane to the retina as follows:

$$
S=T_{V} R_{C L} T_{C L-I C L} R_{I C L} T_{I C L-C o r} R_{C o r} T_{S} R_{S}
$$

where $T_{V}$ represents the translation through the vitreous body, $R_{C L}$ represents the refraction on the crystalline lens, $T_{C L-I C L}$, the translation between the ICL and crystalline lens, $R_{I C L}$, the refraction produced by the ICL, $T_{I C L-C o r}$, the translation between the cornea and ICL, $R_{C o r}$, the refraction on the cornea, $T_{S}$, the translation between the spectacle plane and the cornea, and $R_{S}$, the refraction on the spectacle plane. For a fully corrected optical system, when the incident rays are parallel to the optical axis, they should intercept the optical axis at the position where the image plane (retina) coincides with the optical axis. In the above optical system, $S$, this is achieved when the matrix coordinate on the second row and the second column is equal to zero.

Initially, a series of spectacle lens refractions $( \pm 0.00,-2.00,-6.00,-10.00,-14.00$, $-18.00)$ DS were defined, by means of a single refractive surface, to $12.00 \mathrm{~mm}$ of the corneal plane to create a set of myopic eyes by allowing the elongation of the vitreous body length. Secondly, two refractive surfaces were introduced into the anterior chamber to simulate the presence of an ICL for the correction of myopia. The two surfaces were separated by a constant value of $230 \mu \mathrm{m}$ (ICL thickness) with a refractive index in the optical media between them of 1.442 , representative of the ICL material $(0.2 \%$ collagen and $60 \%$ hydroxylethyl methacrylate) [18]. The ICL power for the correction of each myopic eye was determined by removing the ophthalmic lens, setting the ICL posterior surface radius of curvature as a variable, and solving the $S$ matrix for emmetropia (Equation (4)). The power of the ICL was determined by assuming a $500 \mu \mathrm{m}$ distance between the posterior surface of the ICL and the anterior crystalline lens surface. Thirdly, to investigate the effect of vault size on the postoperative refractive error, the ICLs surfaces were displaced together to simulate the following vault conditions: $0,250,500,750,1000$ and $1500 \mu \mathrm{m}$. The power of a single refractive surface on the spectacle plane $(12.0 \mathrm{~mm}$ from the cornea) was calculated by solving Equation (4) in a reverse order, to calculate the refraction matrix $R_{S}$ corresponding to refraction at the spectacle plane, i.e., the postoperative simulated refraction.

Additionally, we investigated the effect of a lack of agreement between the vertex distance, effectively used during subjective refraction, and the vertex distance inputted for ICL power calculation. Firstly, the spectacle refractions measured at $12.0 \mathrm{~mm}( \pm 0.00$, $-2.00,-6.00,-10.00,-14.00$, and -18.00 DS) were now calculated for different vertex distances $(9.0,10.0,11.0,12.0,13.0,14.0$, and $15.0 \mathrm{~mm})$ and the matrix $S$ was solved to calculate the vitreous body elongation for the new spectacle refractions but placed at $12.0 \mathrm{~mm}$ vertex distance. Secondly, the ICL powers for the correction of the myopic eyes (new spectacle refraction placed at $12.0 \mathrm{~mm}$ vertex distance) were calculated as described previously. Finally, for each level of myopia, the matrix S (Equation (4)) was solved in reverse order, using the ICL power previously calculated, and the axial length determined using the correct myopia, i.e. measured at $12.0 \mathrm{~mm}$ vertex distance. The refraction matrix $R_{S}$ indicated the postoperative simulated refraction as the result of an incorrect vertex distance. 


\section{Results \\ 3.1. Clinical Data}

Table 2 shows the pre- and postoperative refractive and anatomical data of the 73 eyes analyzed (45 were women) for two groups of myopic eyes, both LMG and HMG.

Table 2. Pre- and postoperative refractive and anatomical data. Values are represented by the mean, standard deviation (SD) and range; corrected distance VA (CDVA), central corneal thickness (CCT), internal anterior chamber depth (ACD), white-to-white distance (WTW), and uncorrected distance VA (UDVA).

\begin{tabular}{|c|c|c|c|c|c|c|}
\hline \multirow[b]{2}{*}{ Parameter } & \multicolumn{2}{|c|}{$\begin{array}{c}\text { All } \\
(n=73)\end{array}$} & \multicolumn{2}{|c|}{$\begin{array}{l}\text { Low Myopia: ICL > }-9.5 \text { DS } \\
(n=34)\end{array}$} & \multicolumn{2}{|c|}{$\begin{array}{l}\text { High Myopia: } \mathrm{ICL} \leq-9.5 \text { DS } \\
(n=39)\end{array}$} \\
\hline & Mean \pm SD & Range & Mean \pm SD & Range & Mean \pm SD & Range \\
\hline Age (years) & $32.8 \pm 8.7$ & 18,50 & $34.8 \pm 9.0$ & 20,50 & $31.1 \pm 8.1$ & 18,50 \\
\hline \multicolumn{7}{|c|}{ Preoperative } \\
\hline \multicolumn{7}{|c|}{ Preoperative Refraction (Trail-frame ) } \\
\hline Sphere (DS) & $-8.67 \pm 2.93$ & $-19.00,-2.25$ & $-6.34 \pm 1.40$ & $-8.50,-2.25$ & $-10.71 \pm 2.33$ & $-19.00,-8.00$ \\
\hline Cylinder (DC) & $-0.16 \pm 0.26$ & $-0.75,0.00$ & $-0.19 \pm 0.28$ & $-0.75,0.00$ & $-0.14 \pm 0.24$ & $-0.75,0.00$ \\
\hline $\begin{array}{l}\text { Spherical } \\
\text { Equivalent (DS) }\end{array}$ & $-8.76 \pm 2.90$ & $-19.00,-2.63$ & $-6.43 \pm 1.3$ & $-8.50,-2.63$ & $-10.78 \pm 2.32$ & $-19.00,-8.00$ \\
\hline $\begin{array}{l}\text { CDVA } \\
(\log M A R)\end{array}$ & $0.07 \pm 0.11$ & $0.0,0.40$ & $0.04 \pm 0.05$ & $0.00,0.40$ & $0.12 \pm 0.16$ & $0.0,0.70$ \\
\hline \multicolumn{7}{|l|}{ Biometry } \\
\hline $\mathrm{CCT}(\mu \mathrm{m})$ & $531.8 \pm 33.2$ & $473.0,610.0$ & $518.1 \pm 27.6$ & $479.0,582.0$ & $543.8 \pm 35.1$ & $473.0,610.0$ \\
\hline $\begin{array}{l}\text { Mean Sim K } \\
\text { (DS) }\end{array}$ & $44.2 \pm 1.5$ & $40.30,47.6$ & $44.2 \pm 1.4$ & $40.3,46.6$ & $44.2 \pm 1.7$ & $40.3,47.6$ \\
\hline \multicolumn{7}{|l|}{ Corneal } \\
\hline $\begin{array}{l}\text { Astigmatism } \\
\text { (DC) }\end{array}$ & $-0.66 \pm 0.39$ & $-1.70,0.00$ & $-0.69 \pm 0.38$ & $-1.70,0.00$ & $-0.64 \pm 0.40$ & $-1.50,0.00$ \\
\hline WTW (mm) & $12.1 \pm 0.3$ & $11.4,12.8$ & $12.1 \pm 0.3$ & $11.5,12.8$ & $12.1 \pm 0.3$ & $11.4,12.8$ \\
\hline $\mathrm{ACD}(\mathrm{mm})$ & $3.26 \pm 0.24$ & $2.80,3.80$ & $3.25 \pm 0.23$ & $2.80,3.80$ & $3.27 \pm 0.25$ & $2.84,3.80$ \\
\hline ICL Power (DS) & $-9.63 \pm 2.86$ & $-18.00,-3.00$ & $-7.26 \pm 1.46$ & $-9.00,-3.00$ & $-11.69 \pm 2.06$ & $-18.00,-9.50$ \\
\hline \multicolumn{7}{|c|}{ Postoperative Predicted Refraction } \\
\hline Sphere (DS) & $-0.02 \pm 0.25$ & $-0.48,+0.88$ & $\pm 0.00 \pm 0.26$ & $-0.38,+0.88$ & $-0.03 \pm 0.24$ & $-0.48,+0.67$ \\
\hline Cylinder (DC) & $-0.16 \pm 0.21$ & $-0.71,0.00$ & $-0.18 \pm 0.24$ & $-0.71, \pm 0.00$ & $-0.14 \pm 0.18$ & $-0.56, \pm 0.00$ \\
\hline $\begin{array}{l}\text { Spherical } \\
\text { Equivalent (DS) }\end{array}$ & $-0.10 \pm 0.22$ & $-0.55,+0.68$ & $-0.09 \pm 0.22$ & $-0.50,+0.68$ & $-0.10 \pm 0.22$ & $-0.55,+0.67$ \\
\hline \multicolumn{7}{|c|}{ Postoperative } \\
\hline $\begin{array}{l}\text { Follow-Up } \\
\text { (days) }\end{array}$ & $99 \pm 36$ & $41-366$ & $105 \pm 23$ & 76,167 & $99 \pm 62$ & $41-366$ \\
\hline Vault $(\mu \mathrm{m})$ & $641.9 \pm 303.7$ & $120.0,1490.0$ & $590.3 \pm 320.4$ & 120,1490 & $686.9 \pm 284.9$ & $170.0,1270.0$ \\
\hline \multicolumn{7}{|c|}{ Postoperative Manifest Refraction } \\
\hline Sphere (DS) & $\pm 0.15 \pm 0.38$ & $-0.75,+1.25$ & $0.00 \pm 0.34$ & $-0.75,+0.62$ & $+0.29 \pm 0.37$ & $-0.37,+1.25$ \\
\hline Cylinder (DC) & $-0.35 \pm 0.25$ & $-1.00, \pm 0.00$ & $-0.31 \pm 0.26$ & $-1.00, \pm 0.00$ & $-0.38 \pm 0.24$ & $-1.00,0.00$ \\
\hline $\begin{array}{l}\text { Spherical } \\
\text { Equivalent (DS) }\end{array}$ & $-0.02 \pm 0.37$ & $-0.88,+0.75$ & $-0.16 \pm 0.36$ & $-0.88,+0.50$ & $+0.09 \pm 0.35$ & $-0.63,+0.75$ \\
\hline $\begin{array}{l}\text { UDVA } \\
\text { (logMAR) }\end{array}$ & $0.03 \pm 0.08$ & $-0.08,0.30$ & $0.02 \pm 0.05$ & $-0.08,0.30$ & $0.06 \pm 0.13$ & $-0.08,0.50$ \\
\hline \multicolumn{7}{|c|}{ Postoperative Manifest-Predicted Refraction } \\
\hline $\begin{array}{l}\text { Spherical } \\
\text { Equivalent (DS) }\end{array}$ & $+0.07 \pm 0.33$ & $-0.99,+0.80$ & $+0.07 \pm 0.34$ & $-0.99,+0.53$ & $+0.19 \pm 0.28$ & $-0.25,+0.80$ \\
\hline
\end{tabular}

Comparison between the two myopic groups, using Mann-Whitney tests applied individually to the variables, revealed statistically significant differences between them (Bonferroni correction $(0.05 / 21)=0.0024)$. As expected from the group segmentation, spectacle refraction sphere and spectacle SE and ICL power differed between the two groups $(p<0.001)$. Anatomically, the HMG had, on average, $25.7 \pm 8.53 \mu \mathrm{m}$-thicker corneas than the LMG $(p<0.002)$. Preoperative CDVA was approximately one logMAR 
VA line $(0.08 \pm 0.08 \log$ MAR) lower in the LMG $(p=0.013)$. Postoperatively, the HMG had a tendency toward a more hyperopic refraction, difference $+0.25 \pm 0.08 \mathrm{D}$, compared to the LMG ( $p=0.035)$ (Figure 2a). Despite the fact that these last two comparisons did not achieve the statistical significance limit imposed by the Bonferroni correction, the differences have clinical significance. Nearly $65 \%$ and $54 \%$ of the eyes in the LMG and HMG, respectively, had postoperative refraction within \pm 0.25 D. However, $30 \%$ of the eyes in the HMG had a postoperative SE of between $>+0.25$ and $+1.00 \mathrm{D}$, compared to only $8.8 \%$ in the LMG. In the opposite direction, $26.4 \%$ of the eyes in the LMG had a postoperative SE of between -1.00 and $<-0.25 \mathrm{D}$, compared to $15.4 \%$ in the HMG. Both groups had a nearly unitary relationship between achieved and attempted spherical equivalents (LMG: achieved SE $=1.02 \times($ attempted SE) $+0.20 ;$ HMG: achieved SE $=1.00$ $\times($ attempted SE) -0.29$)$ (Figure $2 b)$.

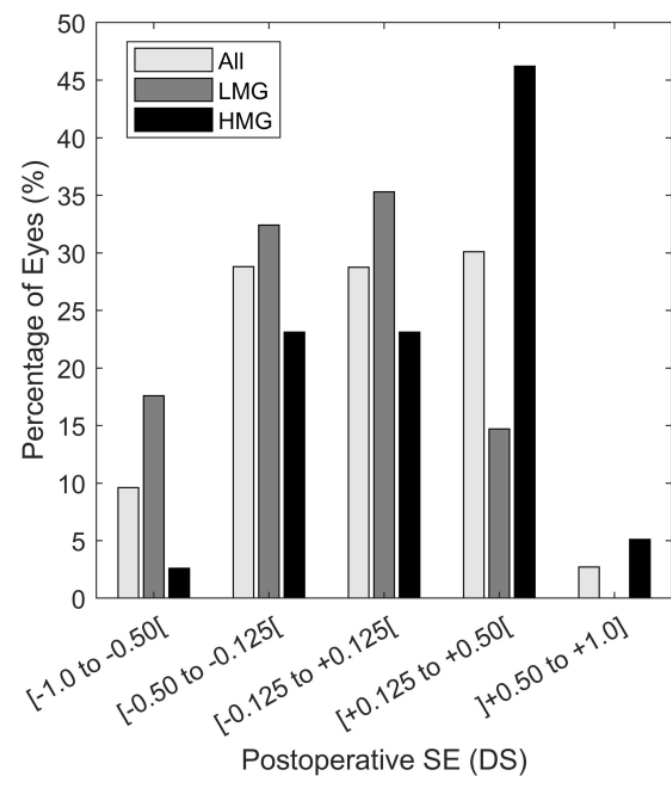

(a)

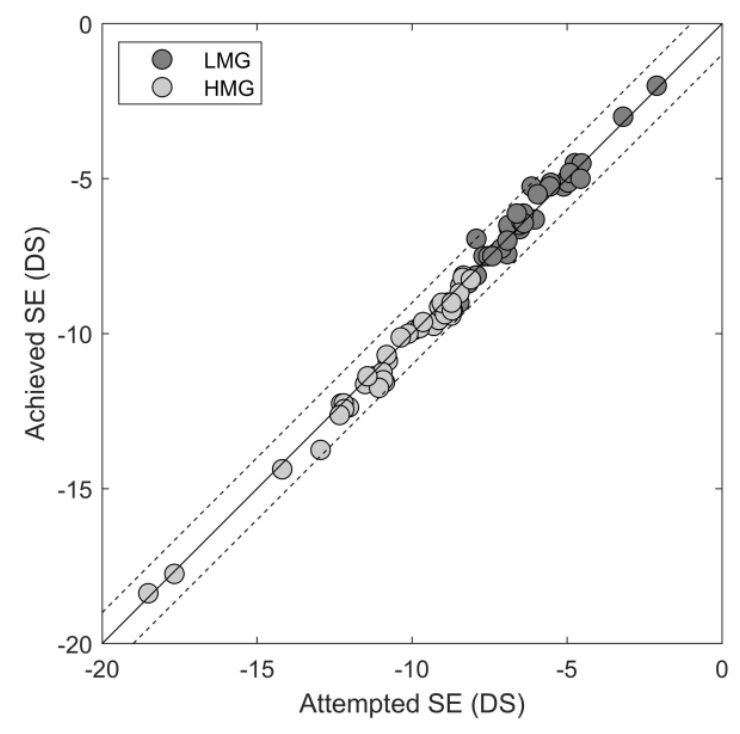

(b)

Figure 2. (a) Postoperative refraction distribution for all cases, in LMG and HMG groups. (b) Association between attempted and achieved SE. Continuous lines and dashed lines represent respectively the unitary relation and \pm 0.50 DS of difference between attempted and achieved SE.

The overall postoperative UDVA was $0.03 \pm 0.08 \log M A R$, with both groups achieving similar VA levels $(p=0.142) ; 91.8 \%$ of the eyes achieved an UDVA of $0.1 \operatorname{logMAR}(20 / 25)$ and $68.5 \%, 0.00 \log$ MAR $(20 / 20)$ (Figure 3a). The efficacy indexes for the LMG and HMG were $1.04 \pm 0.15$ (range: 0.99 to 1.10 ) and $1.13 \pm 0.15$ (range: 1.08 to 1.18$)(p=0.035$ ), respectively, which demonstrates the higher gain in VA associated with ICL surgery, particularly in the HMG. Nearly $60 \%$ of the eyes in the HMG gained more than one VA line compared with $38.2 \%$ of the eyes in the LMG (Figure $3 b$ ).

\subsection{Association between Postoperative Refraction with Demographic, Anatomical, Refractive or ICL-Related Factors}

An exploratory bivariate analysis was conducted, seeking explanatory variables of the postoperative refraction. Demographic, refractive, anatomical, and ICL-related parameters were correlated with the postoperative SE, computed as the difference between the postoperative manifest and predicted SE (Table 3). 


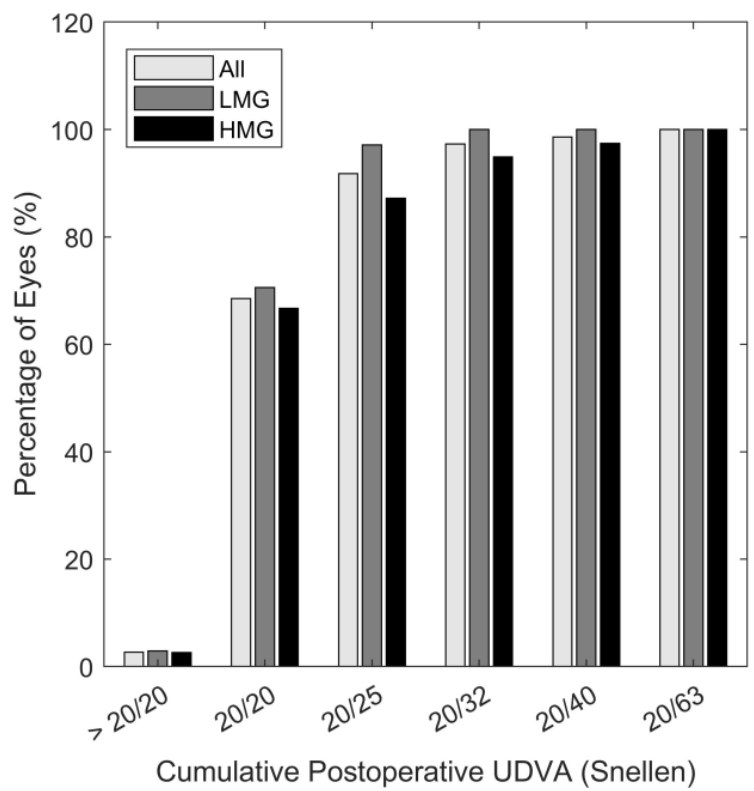

(a)

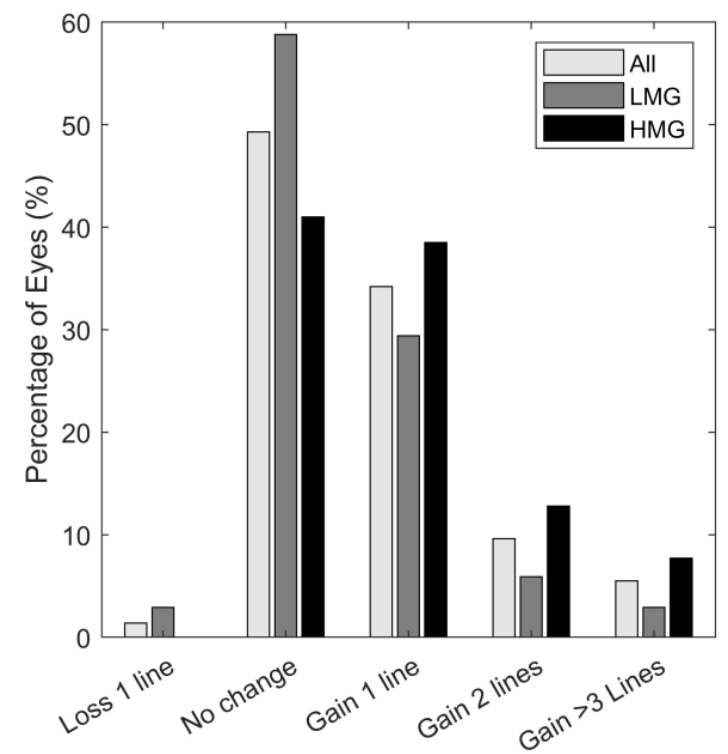

Change in Preoperative CDVA to Postoperative UDVA (Snellen)

(b)

Figure 3. (a) Cumulative percentage of postoperative UCVA in logMAR. (b) Visual acuity variation from preoperative CDVA.

Table 3. Bivariate correlation analysis between postoperative SE and demographic, refractive, anatomical, and ICL-related parameters.

\begin{tabular}{|c|c|c|c|}
\hline $\begin{array}{l}\text { Correlation between } \\
\text { Postoperative SE } \S \text { vs. }\end{array}$ & $\begin{array}{c}\text { All } \\
(n=73)\end{array}$ & $\begin{array}{c}\text { Low Myopia: ICL }>-9.5 \text { DS } \\
(n=34)\end{array}$ & $\begin{array}{l}\text { High Myopia: ICL } \leq-9.5 \text { DS } \\
\qquad(n=39)\end{array}$ \\
\hline Age (years) & $\mathrm{R}=-0.071 p=0.553$ & $\mathrm{R}=0.002 p=0.992$ & $\mathrm{R}=0.089 p=0.589$ \\
\hline $\begin{array}{l}\text { Preoperative Spherical } \\
\text { Equivalent (DS) }\end{array}$ & $\mathrm{R}=-0.367 p=0.001^{*}$ & $\mathrm{R}=-0.217 p=0.218$ & $\mathrm{R}=-0.027 p=0.871$ \\
\hline Central Corneal Thickness $(\mu \mathrm{m})$ & $\mathrm{R}=0.205 p=0.081$ & $\mathrm{R}=0.289 p=0.098$ & $\mathrm{R}=-0.078 p=0.639$ \\
\hline Keratometry (DS) & $\mathrm{R}=0.209 p=0.058$ & $\mathrm{R}=0.111 p=0.533$ & $\mathrm{R}=0.313 p=0.052$ \\
\hline Corneal Cylinder (DC) & $\mathrm{R}=-0.091 p=0.445$ & $\mathrm{R}=-0.068 p=0.704$ & $\mathrm{R}=-0.160 p=0.330$ \\
\hline White-to-White Distance (mm) & $\mathrm{R}=0.181 p=0.126$ & $\mathrm{R}=-0.105 p=0.556$ & $\mathrm{R}=0.029 p=0.860$ \\
\hline Anterior Chamber Depth (mm) & $\mathrm{R}=-0.023 p=0.845$ & $\mathrm{R}=0.011 p=0.952$ & $\mathrm{R}=0.311 p=0.054$ \\
\hline ICL Power (DS) & $\mathrm{R}=-0.382 p=0.001 *$ & $\mathrm{R}=-0.230 p=0.191$ & $\mathrm{R}=-0.091 p=0.584$ \\
\hline Vault $(\mu \mathrm{m})$ & $\mathrm{R}=0.408 p=0.001 *$ & $\mathrm{R}=0.410 p=0.016^{*}$ & $\mathrm{R}=0.356 p=0.026^{*}$ \\
\hline
\end{tabular}

$\S$ Postoperative SE=postoperative manifest SE-postoperative predicted SE; * statistical significance.

The postoperative SE was positively associated with vault magnitude $(R=0.408)$ indicating that eyes with higher vaults show a tendency toward more hyperopic postoperative refraction, compared to eyes with lower vaults. This association was observed in both myopic groups (LMG: $\mathrm{R}=0.410$ and HMG: $\mathrm{R}=0.356$ ), with a postoperative $\mathrm{SE}$ variation of $0.04 \mathrm{D}$ per $100 \mu \mathrm{m}$ of variation in the vault, similar for both groups (univariate ANOVA, $p>0.05$ ) (Figure $4 \mathrm{a}$ ). In addition, the postoperative SE was negatively associated with the spectacle SE $(R=-0.367)$ and ICL power $(R=-0.382)$, indicating that more myopic eyes tended to have more hyperopic postoperative refraction (Figure $4 \mathrm{~b}$ ). The postoperative refraction varied by approximately $0.04 \mathrm{D}$ per diopter of ICL power. 


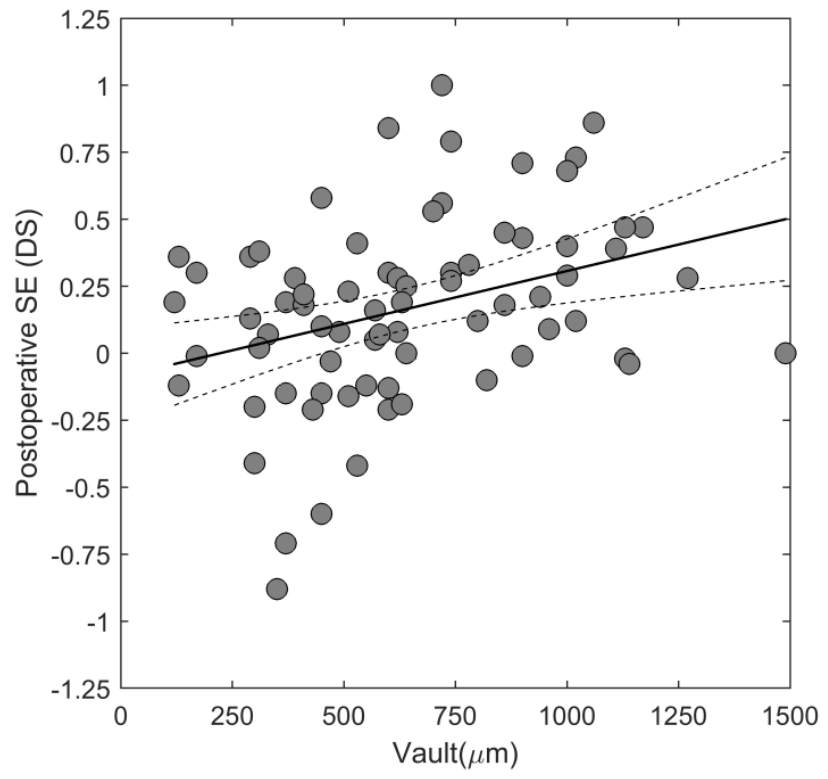

(a)

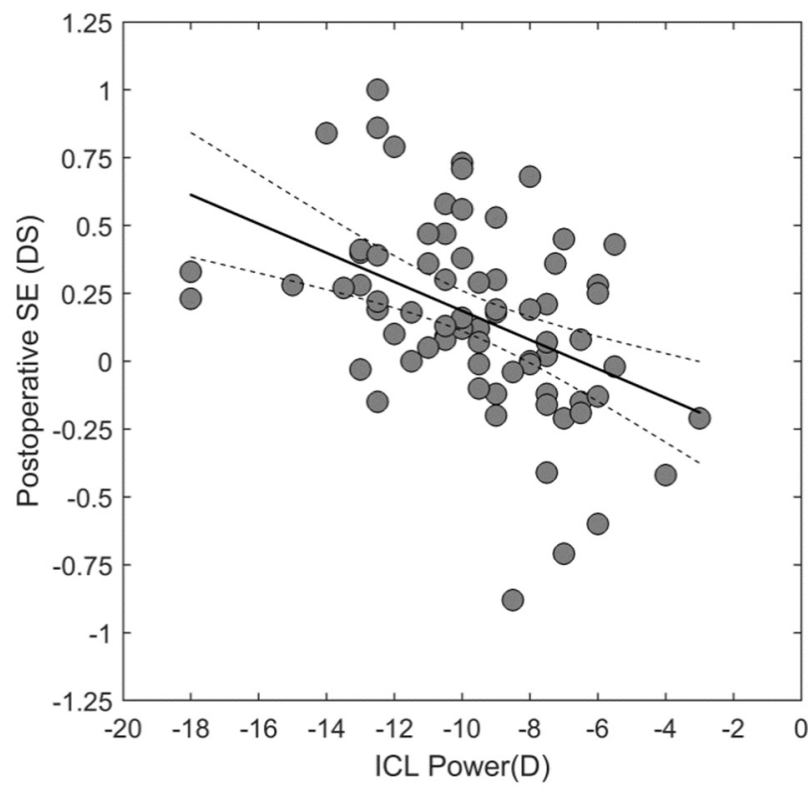

(b)

Figure 4. Association between the postoperative SE and (a) vault, and (b) ICL power. The continuous line represents the linear model fit to the data and the dashed lines represent the $95 \%$ confidence intervals limit. Postoperative SE = postoperative manifest SE - postoperative predicted SE.

\subsection{Paraxial Ray-Tracing}

The observed associations between postoperative refraction, vault and ICL power were investigated through paraxial ray-tracing calculations based on two hypotheses, (1) the intraocular position of the ICL influences the postoperative manifest refraction and (2) a disparity between the vertex distance used during preoperative refraction and the vertex distance inputted for ICL power calculation influences the postoperative manifest refraction. Axial myopia of different magnitudes was obtained by increasing the vitreous body length by $0.391 \mathrm{~mm}$ per diopter (Table 4 ).

Table 4. Shows the parameters used to generate a series of myopic model eyes. Refractive Error, Vitreous Body Length, ICL Posterior Radius Curvature are defined in the model eye as variables.

\begin{tabular}{|c|c|c|c|c|}
\hline \multirow{2}{*}{$\begin{array}{c}\text { Refractive Error } \\
\text { Spectacle Plane (DS) } \\
12.0 \mathrm{~mm}\end{array}$} & \multirow{2}{*}{$\begin{array}{l}\text { Vitreous Body Length } \\
(\mathrm{mm})\end{array}$} & \multirow{2}{*}{$\begin{array}{l}\text { Eye Axial Length } \\
(\mathrm{mm})\end{array}$} & \multicolumn{2}{|c|}{ ICL } \\
\hline & & & $\begin{array}{l}\text { Posterior Radius of } \\
\text { Curvature }(\mathrm{mm})\end{array}$ & Power (D) \\
\hline 0 & 16.66 & 24.2 & -3787.3 & 0.03 \\
\hline-2.00 & 17.34 & 24.9 & 4.41 & -2.37 \\
\hline-6.00 & 18.86 & 26.5 & 1.54 & -6.78 \\
\hline-10.00 & 20.43 & 28.0 & 0.97 & -10.78 \\
\hline-14.00 & 22.04 & 29.6 & 0.73 & -14.33 \\
\hline-18.00 & 23.68 & 31.3 & 0.59 & -17.58 \\
\hline
\end{tabular}

For an ICL calculated to a predetermined intraocular position (e.g., vault $=500 \mu \mathrm{m}$ ) any variation from this position produces a change in the postoperative simulated refraction, with a magnitude of variation depending on the ICL power (Figure 5a). For the optimal vault range, i.e., vault $>250$ and $<750 \mu \mathrm{m}$, the postoperative simulated refraction ranges from \pm 0.03 DS for a -2.00 DS ICL to \pm 0.20 DS when a -18.00 DS ICL is used. The variation in the postoperative simulated refraction per $100 \mu \mathrm{m}$ of vault shift is $0.04 \mathrm{DS}$ for a $-6.0 \mathrm{DS}$, 0.06 DS for a -10.0 DS, and 0.08 DS for a -18.0 DS ICL. 


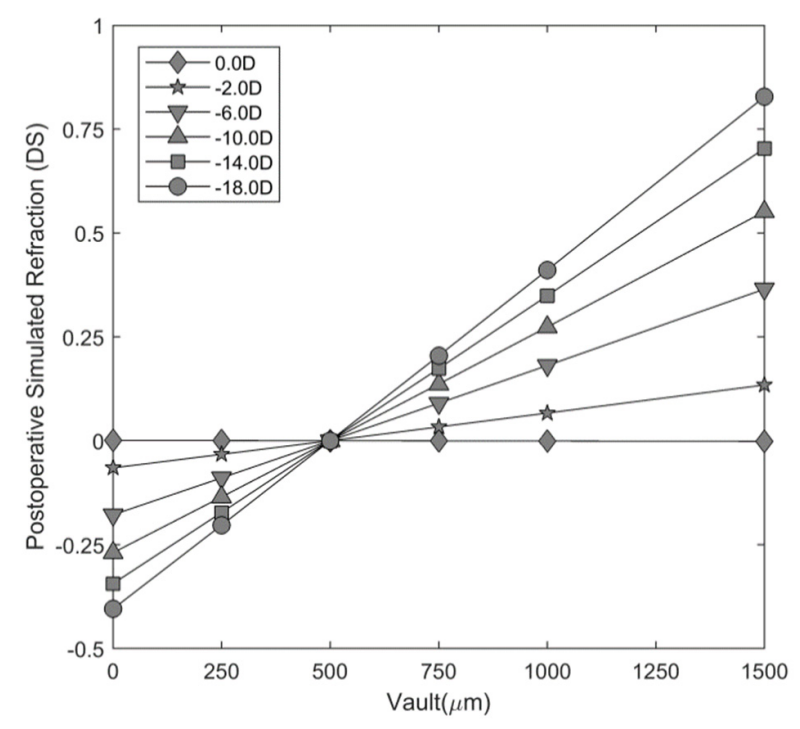

(a)

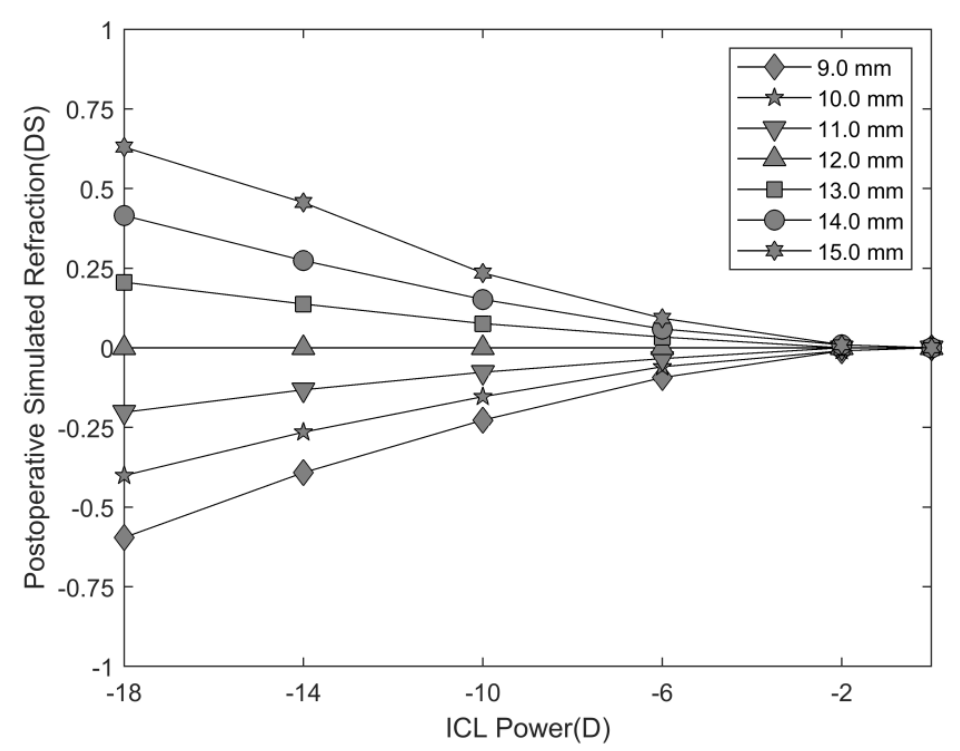

(b)

Figure 5. (a) Postoperative simulated refraction prediction as a function of vault size, for a series of myopic ICLs. The symbols represent the following refractive conditions at the spectacle plane $(\bullet: 0.00$ DS, $\star$ : -2.00 DS, $\mathbf{v}:-6.00$ DS, $\mathbf{\Delta}:-10.00$ DS,

$\mathbf{\square}:-14.00$ DS, $\bullet$ : -18.00 DS). (b) Postoperative simulated refraction prediction as a function of ICL power for various cornea-to-spectacle plane distances (vertex distance). ICL power calculation was performed for a set of refractive powers: 0.00 DS, -2.00 DS, -6.00 DS, -10.00 DS, $-14.00 \mathrm{DS}$ and $-18.00 \mathrm{DS}$, for a vertex distance of $12.0 \mathrm{~mm}$. The symbols represent the following vertex distances used for refraction $(\bullet: 9.0 \mathrm{~mm}, \star: 10.0 \mathrm{~mm}, \mathbf{\nabla}: 11.0 \mathrm{~mm}, \mathbf{\Delta}: 12.0 \mathrm{~mm}, \mathbf{\square}: 13.0 \mathrm{~mm}, \bullet: 14.0 \mathrm{~mm}$, *: $15.0 \mathrm{~mm})$.

The effect of a disparity between the vertex distance inputted for ICL power calculation and the vertex distance used during preoperative refraction was investigated, by predicting the postoperative simulated refraction when the vertex distance used during refraction differed from the predefined $12.0 \mathrm{~mm}$ inputted for ICL power calculation (Figure $5 \mathrm{~b}$ ). In myopic eyes, preoperative refraction, measured at a farther vertex distance than the one used for ICL power calculation, leads to postoperative hyperopic refraction, and leads to myopic postoperative refraction if the vertex distance is shorter than the one used for ICL power calculation. For a constant difference in vertex distance across different myopic powers, the postoperative refraction predicted variation ranges from 0.01 to 0.04 DS per diopter of spherical error when the vertex distance varies between 1.0 and $3.0 \mathrm{~mm}$ around $12.0 \mathrm{~mm}$.

\section{Discussion}

The present study evaluated the refractive predictability and efficacy index of ICL implantation in the correction of myopia. The results confirmed that ICL is a predictable and effective method for the correction of different levels of myopia. Additionally, we investigated the association between postoperative manifest refraction, with optical aspects related to the ICL calculation, and its intraocular position. The clinical findings concur with the ray-tracing predictions, pointing toward a variation in the postoperative manifest refraction associated with the vault. Additionally, we argue that the dependency between postoperative manifest refraction and the ICL power is related to disparities between the vertex distance used during preoperative refraction and that used for ICL power calculation.

The mean postoperative manifest SE for the whole group was close to zero, with the SE extending approximately \pm 0.75 DS. Alfonso et al. found similar outcomes for the same follow-up period for a wide range of myopic eyes [19]. Kamiya et al. analyzed the postoperative results of two groups of myopes, one with myopia of less than -6.00 DS and another of more than -6.00 DS. At three months, the predictability of the postoperative 
refraction was similar between the two groups, with $91 \%$ of the eyes within \pm 0.50 DS and $99 \%$ within \pm 1.00 DS. These results compare to ours, showing that $87.7 \%$ and $100 \%$ of the eyes were within \pm 0.50 and \pm 1.00 DS, respectively [12]. Dividing the group according to ICL power showed poor predictability in the HMG, with $54 \%$ of the eyes in the \pm 0.25 DS range, against $65 \%$ in the LMG. In addition, the LMG showed a tendency toward more myopic postoperative manifest refraction compared to a hyperopic tendency in the HMG. Yan et al., in a group of highly myopic eyes, reported a mean postoperative SE of -0.90 DS, contrasting with the hyperopic tendency observed in our HMG [20]. One factor accounting for this difference may be the number of eyes with myopia over -18.00 and the higher amount of residual astigmatism in Yan's study. The efficacy index of the ICL implantation found in this study $(1.09 \pm 0.16)$ was similar to that reported by Shimizu et al. in a group of myopes ( -3.50 to -11.75 DS) who were implanted with the same ICL version [21]. The efficacy index of ICL implantation was higher in the HMG compared to the LMG (LMG: 1.04 vs. HMG: 1.13), reflected by a higher preoperative CDVA in the LMG, and similar postoperative UCVA between groups. These results concur with the study by Kamyia et al., where the more myopic group showed higher effectiveness [12]. The higher gain in visual acuity observed in highly myopic eyes may be associated with the larger increase in the retinal image in eyes with higher myopia when implanted with phakic IOLs [22]. These results add evidence regarding the effectiveness and predictability of ICL surgery in a wide range of myopias, with additional perceptual advantages in high myopias.

Despite the low postoperative manifest refraction being similar to that reported in other studies, we identified an association between the postoperative SE (postoperative manifest SE minus postoperative predicted SE) and the vault and ICL power. Regarding the association between postoperative SE and the vault, ray-tracing predictions indicate that an increase in the vault from that assumed in ICL power calculation (e.g., $500 \mu \mathrm{m}$ ) produces a postoperative hyperopic refraction and myopic postoperative refraction, if the ICL stands closer to the crystalline lens. This effect is dependent on the ICL power, with more powerful ICLs being more susceptible to inducing changes in postoperative refraction. The simulated range of variation changes between 0.08 DS and 0.04 DS per $100 \mu \mathrm{m}$ of vault change, for -18.0 DS and -6.0 DS ICL, respectively. In our sample, the postoperative SE varied by 0.04 DS per $100 \mu \mathrm{m}$ for an average ICL power of $\sim-9.50 \mathrm{DS}$, and this did not differ between the two myopic groups. One factor accounting for differences between the simulations and the clinical data may be the ability of individuals to accommodate in order to compensate for some degree of hyperopic defocus; in addition, the proximity between the average ICL powers in the two myopic groups may have contributed to the similar effect observed in both groups. Kamiya et al. addressed the association between postoperative variations in vault and postoperative refraction in a sample of 75 eyes. They reported a tendency to hyperopic postoperative refraction in eyes presenting higher vaults, but the effect was negligible [10]. A variation in the vault within the optimal range $(250$ to $750 \mu \mathrm{m}$ ) was expected to change the postoperative refraction within the minimal clinical refractive change, i.e., \pm 0.25 DS, which just did not happen with a -18.0 DS ICL. However, this finding changes when the full spectrum of vaults is considered since $\sim 21 \%$ of the eyes reported in the literature had vaults outside the optimal range [9]. In the present study, $31.5 \%$ of the eyes had vaults outside the optimal vault range and, of those, $26 \%$ were above $750 \mu \mathrm{m}$. Furthermore, the intrinsic ICL vault increases with myopic power [23], which contributes to an increased risk of high vault [24]. For instance, a -18.0 DS ICL is expected to induce a postoperative refraction of +0.25 DS for a vault of $\sim 800 \mu \mathrm{m}$ but, for a $900 \mu \mathrm{m}$ vault, a positive hyperopic refraction is expected for a myopic ICL of -10.0 DS or more. This fact assumes special relevance for pre-presbyopic patients, assuming a $0.34 \mathrm{D}$ decay in accommodative amplitude per year of life [25]; +0.25 DS postoperative refraction corresponds to almost one year's loss in accommodative amplitude. Adding to this is the fact that myopic patients implanted with phakic lenses, when observing an object at a near-distance, have to elicit more accommodation than when using spectacles $[13,14]$. The accommodative demand when observing an object at $0.33 \mathrm{~m}$ 
increases from $0.13 \mathrm{D}$ to $0.83 \mathrm{D}$ for an eye implanted with an ICL of $-2.0 \mathrm{DS}$ and $-18.0 \mathrm{DS}$, respectively (Figure S1 in Supplement). If a combination of high myopia, high vault, and poor accommodative amplitude is present, the patient may face difficulties in focusing on near objects. Currently, apart from the manufacturer's sizing method, which uses the WTW and ACD as biometric features for the lens size selection, there are alternative methods that use biometric parameters, such as the anterior chamber width, the sulcus-to-sulcus, and the crystalline lens rise, aiming to estimate the vault $[23,26,27]$. Reinstein et al. [2] showed that when using a sizing technique based on the sulcus-to-sulcus distance, the vault range could be reduced compared to the WTW method; other studies have provided methodologies for narrowing the vault range [8,24]. Ando et al. [28], when using the NK formula [29] and KS formula [30] for predicting the postoperative vault, reported differences between the real and achieved vault of $-74.2 \pm 338.7 \mu \mathrm{m}$ and $-129.4 \pm 264.6$, respectively. Considering the $95 \%$ confidence intervals of the differences, if -18.0 DS (the most myopic ICL) was calculated using the predicted vault, the postoperative refractive error associated with vault error would be lower than 0.40 DS (when using the NK formula). While it is not possible to narrow down the vault error, at the moment of selecting the ICL power, the surgeon should consider the expected vault for the ICL size chosen $(26,27)$ and select the ICL power by combining the predicted postoperative refraction, calculated by the manufacturer, and the expected postoperative refraction, induced by the expected lens position.

Another finding in the present study was the dependency of postoperative SE on the ICL power, indicating that more myopic eyes, i.e., those with more myopic ICL implanted, have a tendency toward more hyperopic postoperative refraction and low myopic ICL, showing, on average, lower postoperative refractions. We advance a possible explanation for this finding based on the disparity between the vertex distance used during refraction and the vertex distance inputted for ICL calculation $(12.0 \mathrm{~mm}$ in all cases). The modified vertex formula used by the manufacturer for ICL power calculation assumes the refraction on the corneal plane, thus requiring a correct translation for the spectacle to the corneal plane [7]. The ray-tracing predictions indicate that the refraction that is performed at a larger vertex distance than the vertex distance used for ICL calculation produces an over-minusing of the ICL correction [11]. Combining the clinical data and the predictions suggests that the vertex distance used for refraction was larger than $12.0 \mathrm{~mm}$. Comparing the variation of postoperative refraction, with an ICL power of $0.04 \mathrm{D}$ per diopter of ICL power, with the simulated variations using the model eye, we could argue that the trial lens(es) should have been placed, on average, at $15.0 \mathrm{~mm}$ in front of the corneal plane. This should be taken merely as a possible explanation for the association between postoperative refraction and ICL power, since no control of the vertex distance took place during the refraction. Since vertex distances have large inter- and intra-individual variations [31], associated with the fact that phakic ICLs are often applied for the correction of high refractive errors, an accurate measure of the vertex distance is of the utmost relevance in this type of procedure. An alternative procedure might involve using a contact lens and performing an over-refraction, which would eliminate the influence of the vertex distance.

This study has a number of limitations. One is in regard to the retrospective nature of the study. Conducting this study prospectively could have allowed us to better control aspects related to postoperative refraction by establishing a standard subjective refraction protocol that could have improved the accuracy of postoperative refraction. A second limitation relates to the influence of the vertex distance, which could have been controlled by canceling out its influence on the postoperative results. A third aspect relates to the variations in the vault with pupil size [32] and its potential influence on postoperative refraction. It is known that light and, to a smaller extent, accommodation [33] produce changes in vault size, but the magnitude of vault variations (light-induced: $167 \mu \mathrm{m}$ [32]) are likely to have little effect on postoperative refraction. On the other hand, the sample size gave the study a power of 0.97 for detecting a variation in the postoperative refraction for -9.5 DS of ICL power. In addition, the ability to support the experimental data with theoretical predictions strengthens the clinical relevance of this study. All these points 
support the need for future research addressing the influence of ICL surgery postoperative refraction on near-vision and the influence of vault predictability on postoperative refraction. In conclusion, this study supports the concept that ICLs are a predictable and effective form of refractive surgery for a wide range of myopias. The accurate control of preoperative refraction, especially the vertex distance and the prediction of the postoperative vault, are variables that can help to improve the refractive predictability of the surgery.

Supplementary Materials: The following are available online at https: / www.mdpi.com/article/10 .3390/opt2040028/s1, Figure S1: Accommodative demand spectacles and phakic ICL.

Author Contributions: The work described in this article results from the collaborative effort of all authors. Conceptualization, P.S., S.C.M. and Á.S.T.; methodology, P.S., S.C.M.; software, P.S.; formal analysis, P.S. and A.B.; investigation, P.S. and O.T.S.; resources, S.C.M. and Á.S.T.; data curation, P.S.; writing-P.S., O.T.S. and A.B.; writing—review and editing, P.S. and O.T.S.; visualization, P.S.; supervision, S.C.M.; project administration, Á.S.T. All authors have read and agreed to the published version of the manuscript.

Funding: This research received no external funding.

Institutional Review Board Statement: The study was conducted according to the guidelines of the Declaration of Helsinki, and approved by the Ethics Committee of Investigación Clínica de Badajoz (Spain) (date of approval: 27/03/2019).

Informed Consent Statement: Patient consent was waived due to the retrospective nature of the study.

Data Availability Statement: The data presented in this study are available on request from the corresponding author. The data are no publicly available due to privacy obligations.

Conflicts of Interest: The authors declare no conflict of interest.

\section{References}

1. Pineda, R.; Chauhan, T. Phakic intraocular lenses and their special indications. JOVR 2016, 11, 422-428. [CrossRef] [PubMed]

2. Reinstein, D.Z.; Lovisolo, C.F.; Archer, T.J.; Gobbe, M. Comparison of postoperative vault height predictability using white-towhite or sulcus diameter-based sizing for the visian implantable collamer lens. J. Refract. Surg. 2013, 29, 30-35. [CrossRef]

3. Packer, M. The Implantable Collamer Lens with a central port: Review of the literature. Clin. Ophthalmol. 2018, 12, 2427-2438. [CrossRef] [PubMed]

4. Ieong, A.; Hau, S.C.H.; Rubin, G.S.; Allan, B.D.S. Quality of life in high myopia before and after implantable Collamer lens implantation. Ophthalmology 2010, 117, 2295-2300. [CrossRef]

5. Atchison, D.A. Refractive errors induced by displacement of intraocular lenses within the pseudophakic eye. Optom. Vis. Sci. 1989, 66, 146-152. [CrossRef]

6. Nawa, Y.; Ueda, T.; Nakatsuka, M.; Tsuji, H.; Marutani, H.; Hara, Y.; Uozato, H. Accommodation obtained per 1.0 mm forward movement of a posterior chamber intraocular lens. J. Cataract Refract. Surg. 2003, 29, 2069-2072. [CrossRef]

7. Holladay, J.T. Refractive power calculations for intraocular lenses in the phakic eye. Am. J. Ophthalmol. 1993, 116, 63-66. [CrossRef]

8. Lee, H.; Kang, D.S.; Choi, J.Y.; Ha, B.J.; Kim, E.K.; Seo, K.Y.; Kim, T.I. Analysis of pre-operative factors affecting range of optimal vaulting after implantation of 12.6-mm V4c implantable collamer lens in myopic eyes. BMC Ophthalmol. 2018, 18, 163. [CrossRef]

9. Packer, M. Meta-analysis and review: Effectiveness, safety, and central port design of the intraocular collamer lens. Clin. Ophthalmol. 2016, 10, 1059-1077. [CrossRef]

10. Kamiya, K.; Shimizu, K.; Kawamorita, T. Changes in vaulting and the effect on refraction after phakic posterior chamber intraocular lens implantation. J. Cataract Refract. Surg. 2009, 35, 1582-1586. [CrossRef]

11. Weiss, R.; Berke, W.; Gottlieb, L.; Horvath, P. Clinical importance of accurate refractor vertex distance measurements prior to refractive surgery. J. Refract. Surg. 2002, 18, 444-448. [CrossRef] [PubMed]

12. Kamiya, K.; Shimizu, K.; Igarashi, A.; Kitazawa, Y.; Kojima, T.; Nakamura, T.; Oka, Y.; Matsumoto, R. Posterior chamber phakic intraocular lens implantation: Comparative, multicentre study in 351 eyes with low-to-moderate or high myopia. Br. J. Ophthalmol. 2018, 102, 177-181. [CrossRef]

13. Langenbucher, A.; Szentmáry, N.; Seitz, B. Magnification and accommodation with phakic intraocular lenses. Ophthalmic Physiol. Opt. 2007, 27, 295-302. [CrossRef]

14. Hernández-Rodríguez, C.J.; Piñero, D.P. A systematic review about the impact of phakic intraocular lenses on accommodation. J. Optom. 2020, 13, 139. [CrossRef] [PubMed]

15. Holladay, J.T. Proper method for calculating average visual acuity. J. Refract. Surg. 1997, 13, 388-391. [CrossRef] 
16. Atchison, D.A.; Smith, G. Optics of the Human Eye; Butterworth-Heinemann: Oxford, UK; Elsevier: Amsterdam, The Netherlands, 2000; p. 288.

17. Langenbucher, A.; Reese, S.; Sauer, T.; Seitz, B. Matrix-based calculation scheme for toric intraocular lenses. Ophthalmic Physiol. Opt. 2004, 24, 511-519. [CrossRef] [PubMed]

18. Sanders, D.R.; Doney, K.; Poco, M. United States Food and Drug Administration clinical trial of the Implantable Collamer Lens (ICL) for moderate to high myopia: Three-year follow-up. Ophthalmology 2004, 111, 1683-1692.

19. Alfonso, J.F.; Baamonde, B.; Fernández-Vega, L.; Fernandes, P.; González-Méijome, J.M.; Montés-Micó, R. Posterior chamber collagen copolymer phakic intraocular lenses to correct myopia: Five-year follow-up. J. Cataract Refract. Surg. 2011, 37, 873-880. [CrossRef] [PubMed]

20. Yan, Z.; Miao, H.; Zhao, F.; Wang, X.; Chen, X.; Li, M.; Zhou, X. Two-Year Outcomes of Visian Implantable Collamer Lens with a Central Hole for Correcting High Myopia. J. Ophthalmol. 2018, 2018, 8678352. [CrossRef] [PubMed]

21. Shimizu, K.; Kamiya, K.; Igarashi, A.; Shiratani, T. Early clinical outcomes of implantation of posterior chamber phakic intraocular lens with a central hole (Hole ICL) for moderate to high myopia. Br. J. Ophthalmol. 2012, 96, 409-412. [CrossRef]

22. García, M.; González, C.; Pascual, I.; Fimia, A. Magnification and visual acuity in highly myopic phakic eyes corrected with an anterior chamber intraocular lens versus by other methods. J. Cataract Refract. Surg. 1996, 22, 1416-1422. [CrossRef]

23. Lee, D.H.; Choi, S.H.; Chung, E.S.; Chung, T.Y. Correlation between preoperative biometry and posterior chamber phakic Visian Implantable Collamer Lens vaulting. Ophthalmology 2012, 119, 272-277. [CrossRef] [PubMed]

24. Cerpa Manito, S.; Sánchez Trancón, A.; Torrado Sierra, O.; Baptista, A.M.; Serra, P.M. Biometric and ICL-related risk factors associated to sub-optimal vaults in eyes implanted with implantable collamer lenses. Eye Vis. 2021, 8, 26. [CrossRef]

25. Mordi, J.A.; Ciuffreda, K.J. Static aspects of accommodation: Age and presbyopia. Vis. Res. 1998, 38, 1643-1653. [CrossRef]

26. Trancón, A.S.; Manito, S.C.; Sierra, O.T.; Baptista, A.M.; Serra, P.M. Determining vault size in implantable collamer lenses: Preoperative anatomy and lens parameters. J. Cataract Refract. Surg. 2020, 46, 728-736. [CrossRef]

27. Kamiya, K.; Ryu, I.H.; Yoo, T.K.; Kim, J.S.; Lee, I.S.; Kim, J.K.; Ando, W.; Shoji, N.; Yamauchi, T.; Tabuchi, H. Prediction of Phakic Intraocular Lens Vault Using Machine Learning of Anterior Segment Optical Coherence Tomography Metrics. Am. J. Ophthalmol. 2021, 226, 90-99. [CrossRef] [PubMed]

28. Ando, W.; Kamiya, K.; Hayakawa, H.; Takahashi, M.; Shoji, N. Comparison of Phakic Intraocular Lens Vault Using Conventional Nomogram and Prediction Formulas. J. Clin. Med. 2020, 9, 4090. [CrossRef]

29. Nakamura, T.; Isogai, N.; Kojima, T.; Yoshida, Y.; Sugiyama, Y. Optimization of implantable collamer lens sizing based on swept-source anterior segment optical coherence tomography. J. Cataract Refract. Surg. 2020, 46, 742-748. [CrossRef]

30. Igarashi, A.; Shimizu, K.; Kato, S.; Kamiya, K. Predictability of the vault after posterior chamber phakic intraocular lens implantation using anterior segment optical coherence tomography. J. Cataract Refract. Surg. 2019, 45, 1099-1104. [CrossRef]

31. Sebag, M.; Meslin, D. Refraction: Vertex Distance Matters. Points de Vue. 2020, pp. 1-5. Available online: https://www. pointsdevue.com/sites/default/files/refraction_vertex_distance_matters.pdf (accessed on 10 December 2021).

32. Gonzalez-Lopez, F.; Mompean, B.; Bilbao-Calabuig, R.; Vila-Arteaga, J.; Beltran, J.; Baviera, J. Dynamic Assessment of LightInduced Vaulting Changes of Implantable Collamer Lens With Central Port by Swept-Source OCT: Pilot Study. Transl. Vis. Sci. Technol. 2018, 7, 4. [CrossRef] [PubMed]

33. Du, C.; Wang, J.; Wang, X.; Dong, Y.; Gu, Y.; Shen, Y. Ultrasound biomicroscopy of anterior segment accommodative changes with posterior chamber phakic intraocular lens in high myopia. Ophthalmology 2012, 119, 99-105. [CrossRef] [PubMed] 